\title{
Ionospheric response to variable electric fields in small-scale auroral structures
}

\author{
B. S. Lanchester ${ }^{1}$, M. H. Rees ${ }^{1}$, K. J. F. Sedgemore ${ }^{1}$, J. R. Palmer ${ }^{1}$, H. U. Frey ${ }^{2^{*}}$, K. U. Kaila ${ }^{3}$ \\ ${ }^{1}$ Department of Physics, University of Southampton, Southampton, SO17 1BJ, UK \\ Fax: + 441703 593910; e-mail: bsl@phys.soton.ac.uk \\ ${ }^{2}$ Max-Planck-Institut für Extraterrestische Physik, D-85740 Garching, Germany \\ ${ }^{3}$ Department of Physics, University of Oulu, FIN-90570 Oulu, Finland
}

Received: 23 January 1998 / Revised: 18 March 1998 / Accepted: 25 March 1998

\begin{abstract}
High time and space resolution optical and radar measurements have revealed the influence of electric fields on E-region electron density profiles in small-scale auroral structures. Large electric fields are present adjacent to auroral filaments produced by monoenergetic electron fluxes. The ionisation profiles measured within and beside the auroral filaments show the effects of plasma convection due to electric fields as well as the consequences of the response time to large and dynamic fluxes of energetic electrons. Without highresolution optical measurements, the interpretation of the radar data is limited.
\end{abstract}

Key words. Auroral ionosphere $\cdot$ Ionospheremagnetosphere interactions $\cdot$ EISCAT

\section{Introduction}

Small-scale spatial structure in optical aurora that has been observed for decades has recently been complemented by in situ measurements on satellites of energetic particle fluxes, electric fields and currents. This development is the result of the high time resolution of the new instruments, which is essential for interpreting data from platforms travelling at speeds of several $\mathrm{km} / \mathrm{s}$. The Freja satellite and the FAST satellite have provided such high spatial resolution measurements (e.g. Carlson et al., 1997; Boehm et al., 1994, 1995). Satellite and rocketborne measurements are acquired as a function of time, but represent, primarily, the spatial variation of the measured parameters. We have used ground-based optical imaging and radar measurements to investigate

Correspondence to: B. S. Lanchester

* Present address: Space Sciences Laboratory, University of California, Berkeley coincident space and time variations in small-scale aurora. In particular, we show the spatial relationship between electric fields derived from tristatic radar data and auroral filaments at one location over time-intervals inaccessible to satellites and rockets. We use a timedependent auroral model to interpret the measurements. The analysis shows the effect of plasma convection due to the large measured electric field. It also highlights the problems associated with interpretation of radar data, due to the response time of the ionosphere to large and dynamic fluxes of electron precipitation.

The auroral model used in the following analysis is described in Lanchester et al. (1994), with further refinements essential to this work described in Lanchester et al. (1997). In the latter, it was found that the incoming flux of electrons in a very bright and narrow $(100 \mathrm{~m})$ auroral filament was made up of a monoenergetic sheet, embedded in a wider region of electron precipitation with a spread of energies. The model was adapted to accept as input the combination of the two fluxes, with different spectral characteristics. The important point made in that work was that the radar measurements of electron density were taken over a field of view that combined both the monoenergetic beam and the surrounding broad spectral distribution. This is found to be the case in the present event, but there are other factors at work as well, which are discovered only by comparison of modelled and measured electron density profiles. Kaila and Rasinkangas (1989) have shown that single Maxwellian or monoenergetic distributions do not explain all observed profiles. Similar results were found by Rasinkangas et al. (1989).

\section{Observations}

The observations used in this work were made at Tromsø, Norway, during an auroral campaign in January 1995. The EISCAT UHF radar experiment (PULSE) was designed to measure the electron density during rapidly varying auroral events, with power 
profiles measured to very high resolution (range $1.05 \mathrm{~km}, 0.2 \mathrm{~s}$ ) in the $\mathrm{E}$ region between 75 and $145 \mathrm{~km}$. The plasma parameters are obtained in the $\mathrm{F}$ region at lower resolution (range $12 \mathrm{~km}, 3 \mathrm{~s}$ ), and tristatic measurements of plasma velocity are made at $278 \mathrm{~km}$. The filter photometer had a field of view of $0.5^{\circ}$ which was coincident with the field-aligned radar beam. The emission used in this work is at wavelength $427.8 \mathrm{~nm}$, but measurements were also made at 630.0, 557.7 and $486.1 \mathrm{~nm}$ (University of Oulu). Two imageintensified CCD cameras were operated at 25 frames/s with fields of view of $86^{\circ} \times 64^{\circ}$ and $15^{\circ} \times 21^{\circ}$ (MaxPlanck-Institute for Extraterrestrial Physics). Both cameras had short-wavelength cut-off filters at $650 \mathrm{~nm}$, thus eliminating the long-lived emissions from atomic oxygen, and allowing the prompt emissions of $\mathrm{N}_{2}^{+}$(Meinel) and $\mathrm{N}_{2} 1 \mathrm{PG}$ bands to produce a very sharp image (Frey et al., 1996). Cameras were also operated by the University of Sussex.

The event described here occurred between 21:05 and 21:07 UT on 30 January, 1995, about 10 min later than another active auroral event analysed in Lanchester $e t$ al. (1997). Between the two events the sky above the radar at Tromsø was without auroral structure and radar measurements showed low values of electron density. All-sky images from Tromsø (P. Smith, private communication) show that a large folded band of aurora remained active in the southern sky, with a very bright bulge forming in the eastern part at 21:03 UT, with the whole length of the arc system in very active state, displaying fast temporal variations along its length. At 21:05 UT, this activity reduced, leaving an east-west extended band on the southern horizon. This band began to move north towards the radar, while at the same time new auroral forms appeared ahead of its motion. The arrival of aurora in the field-aligned radar beam at 21:05:20 UT is seen in the E-region electron density profiles of Fig. 1a, which show a very abrupt increase at this time. The auroral images from the two cameras show that the aurora in the radar beam was a complicated display of fast-changing structure between 21:05:20 and 21:05:50 UT. The wide-angle camera puts this in context, and shows that the field of view of the narrow-angle camera is sampling the structure within the arc system, which has a large fold in its length. The sequence of images from the wide-angle camera in Fig. 2 starts at 21:05:36 UT during this active period; north is to the right and east is at the top. The position of the radar beam is marked with a cross. Following a burst of activity, the arc system, clearly made up of two main bands, retreated south to about $30 \mathrm{~km}$ from the radar beam, leaving the sky in the immediate vicinity of the radar apparently dark. The 'arc' then moved steadily north crossing through the field-aligned position at 21:06:06 UT and continuing to the north. This second passage of an 'arc', which in fact was made up of two main structures, and within which there was much elemental structure, is analysed in greatest detail below. The sequence including two passages of the auroral form over the radar is shown in panels 1-9 of Fig. 2 which are separated by a few seconds.
The second panel of Fig. 1 shows the electric field components for the same 2-min interval 21:0521:07 UT. The vectors are shown at the highest time resolution of $3 \mathrm{~s}$. The errors in these measurements are likely to be large, perhaps $\pm 30 \%$ (Lanchester et al., 1996). However, the time-series demonstrates the large and variable electric fields that are measured close to auroral structures. At the start of the interval the electric field is predominantly pointing to the south, in the direction of the approaching arc system. When the arc reaches the radar (21:05:20 UT), the electric field vectors swing between SE and NW as the structures pass through the beam. A very large SE field is measured at 21:05:42 UT when the arc is extremely bright, with the fold in its length such that the electric field vector points towards the arc. The electric field vector at this time is superimposed on the wide-angle image (Fig. 2, panel 3) corresponding to the middle of the 3-s interval. The arc remained about $10 \mathrm{~km}$ from the radar beam during this measurement. During the next $15 \mathrm{~s}$ the electric field values are close to zero. There is aurora in the beam at this time, which appears to form as a new, fainter fold in the original arc. Although it is less bright, it is still variable and dynamic. During the next $10 \mathrm{~s}$ (from 21:06:00) the fields are again consistently towards the $\mathrm{S}$ and SE where the arc has retreated to a distance of about $30 \mathrm{~km}$, with a brighter section in the SE direction. Once the arc moves back towards the radar and into the beam, the electric field vectors reverse several times until after the arc system has passed through to the north, when the vectors are again consistently to the $\mathrm{N}$ and NW, towards the arc. It must be remembered that during the $3 \mathrm{~s}$ that the electric field is sampled, the aurora can change significantly.

The other measured quantity, the prompt emission at $427.8 \mathrm{~nm}$ from the $(0,1)$ vibrational transition of $\mathrm{N}_{2}^{+}$ $1 \mathrm{NG}$, is shown in the third panel of Fig. 1 for the same 2-min interval. The peak of $50 \mathrm{kR}$ is at 21:05:40 UT during the first passage of the arc structure, and at the time shown in panel 2 (Fig. 2). At this time a brightening in the arc coincides with a folded structure in its length. The changes in the intensity measured by the photometer match exactly the changes seen in the images from the narrow-angle camera, showing the high degree of alignment between the two instruments' fields of view on this campaign. This also emphasises the value of the cut-off filter, which allows only the prompt emissions of $\mathrm{N}_{2}^{+}$(Meinel) and $\mathrm{N}_{2}$ 1PG from wavelengths longer than $650 \mathrm{~nm}$ to be measured by the imager. The second passage of the arc structure seen at 21:06:06 UT is also very bright, with more than $30 \mathrm{kR}$ of $427.8-\mathrm{nm}$ emission. Between the two events the 'background' emission remains quite high at about $8 \mathrm{kR}$.

The first auroral increase at the radar (21:05:20 UT) is much more complex optically in space and time variations. The arc system was undergoing a brightening associated with a fold in its length when it was close to the radar. The second passage of the auroral band was steadier in its behaviour as it moved north. Figure 3 is a composite of images from the narrowangle camera during this second passage. For compar- 

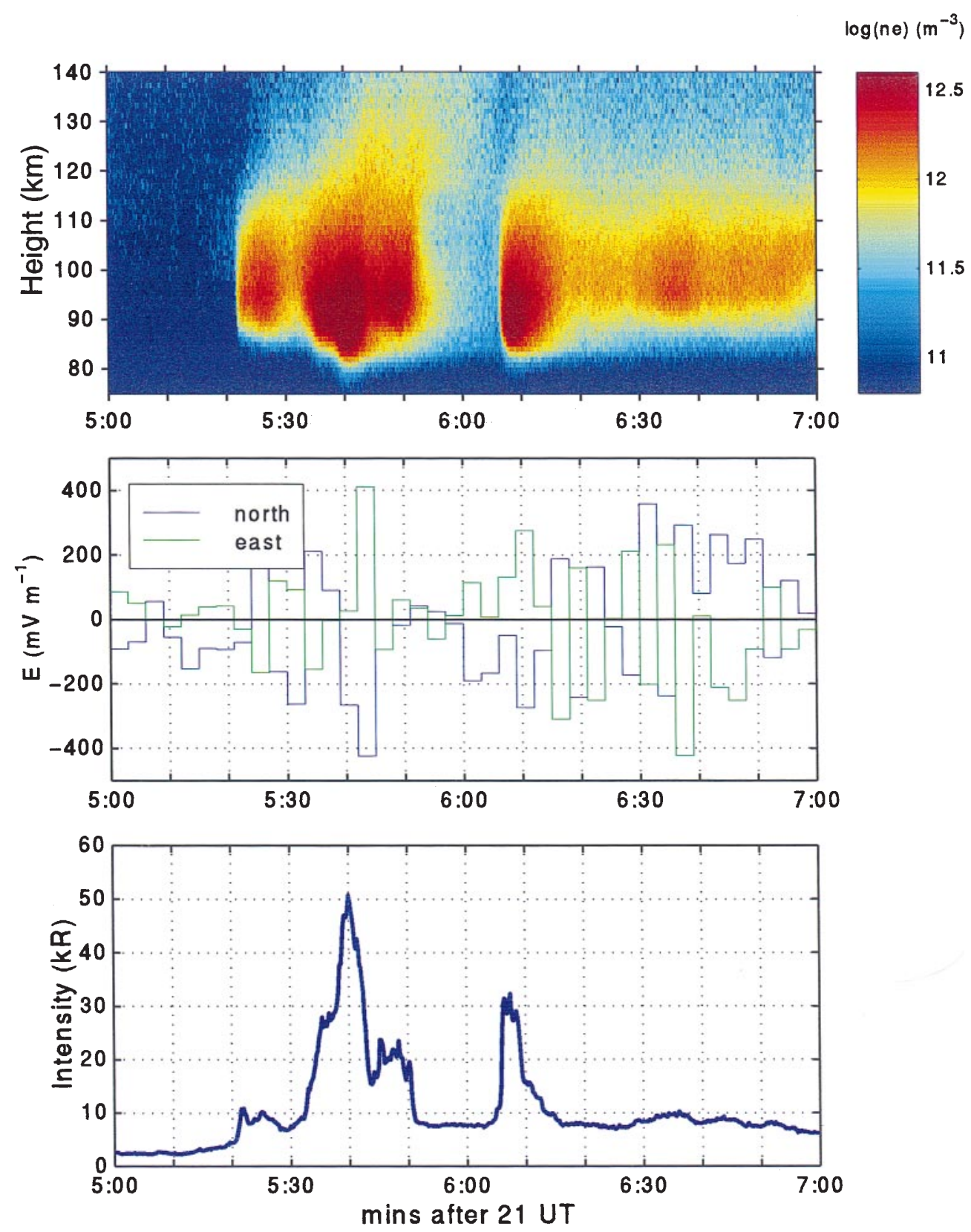

Fig. 1. Top panel: electron density profiles at 0.2 -s resolution showing both passages of the arc system through the field-aligned radar. Middle panel: horizontal electric field components at 3-s resolution measured at $278 \mathrm{~km}$. The largest field at 21:05:42 UT is in SE direction. Bottom panel: intensity of 427.8-nm emission rate in field parallel direction

ison, the last three panels of Fig. 2 show the wide-angle images as the structure moves from south to north (L to R), meeting the radar beam at 21:06:06 UT. The narrow-angle images of Fig. 3 attempt to show the finescale detail that is present within the wider features. This is difficult to achieve on the printed page using still images. Again the position of the radar beam is marked. The two main structures in the arc are seen, but there are also several very narrow elemental structures associated with each of the larger bands. It can be seen that these filaments are narrower than the field of view of the radar and photometer. The arrival of each filament corresponds to an increase in the 427.8-nm intensity.

To see this more clearly, photometer measurements at 0.2 -s resolution, for an interval of only $10 \mathrm{~s}$ between
21:06:04 and 21:06:14 UT, are shown in Fig. 4 a. These data have been corrected for scattering and extinction in the lower atmosphere by applying a correction factor of 1.65 to the measured values. The variations between the peaks and troughs during the passage of the structured arc are seen in the 427.8-nm emission, varying by $\pm 5 \mathrm{kR}$ in less than $1 \mathrm{~s}$. Panel $\mathrm{b}$ gives the detail from the EISCAT electron density profiles at the same time resolution. The peak electron density is $6 \times 10^{12} \mathrm{~m}^{-3}$ and the height of this peak is close to $90 \mathrm{~km}$. The variations in the emission rate are not reflected in the electron density measurements, which are controlled by the ion production and loss rates. For this reason there is also a delay of a fraction of a second in the electron density following the arrival of the auroral bands in the radar beam. 

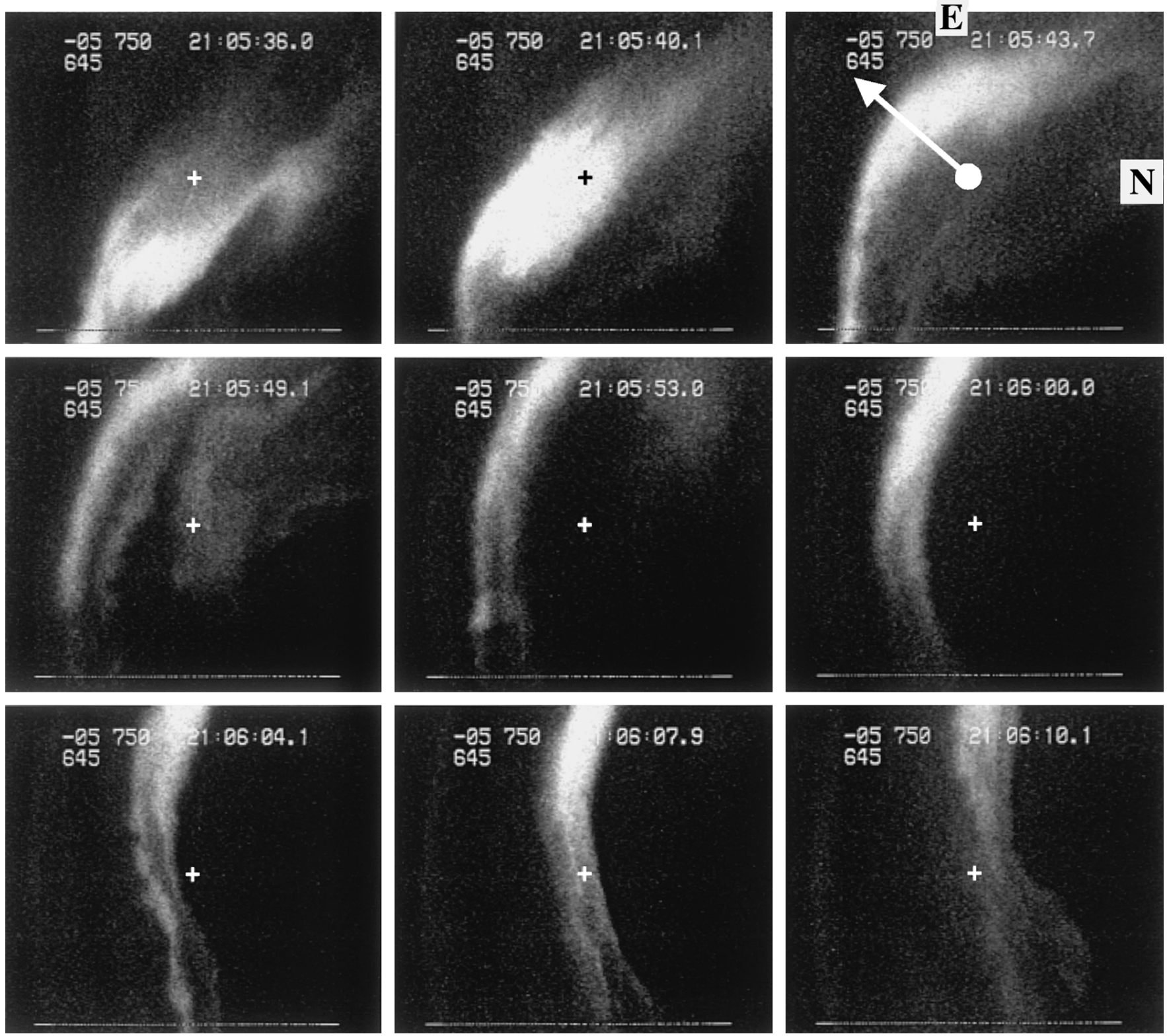

Fig. 2. Sequence of images from wide-angle camera at 21:05:36.0, 40.1, 43.7, 49.1, 53.0, and 21:06:00.0, 04.1, 07.9, 10.1 UT. The position of the radar beam is marked with a cross. North is to the right and

\section{Modelling}

\subsection{General}

One output of the auroral model is a time-history of electron density profiles which can be compared with those measured by the radar. This leads to an indirect method for obtaining the incident energy spectrum. The model solves the electron transport equation, the coupled continuity equations for electrons and all important positive ions and minor neutral species, and the electron and ion energy equations. The input required is an estimate of the shape and peak energy of the electron energy spectrum at each time step, east at the top. The horizontal electric field vector (3-s resolution) superimposed on panel 3 has magnitude $600 \mathrm{mV} \mathrm{m}^{-1}$

and the magnitude of the precipitating energy flux. By fitting the measured electron density profiles to the results of the model, the most appropriate energy distribution is found. As described in Lanchester et al. (1997) the energy of the precipitating flux can be made up of several components, and in the event described therein, the majority of the energy flux resided in a very narrow auroral filament of only $100 \mathrm{~m}$ width, which had a monoenergetic spectrum, represented in the model by a Gaussian distribution with a $10 \%$ half-width. It was embedded in a region with a spread of energies, represented in the model by a Maxwellian spectrum. The resulting electron density profiles from both of these distributions were sampled by the radar at the same time, and therefore required careful interpretation. 

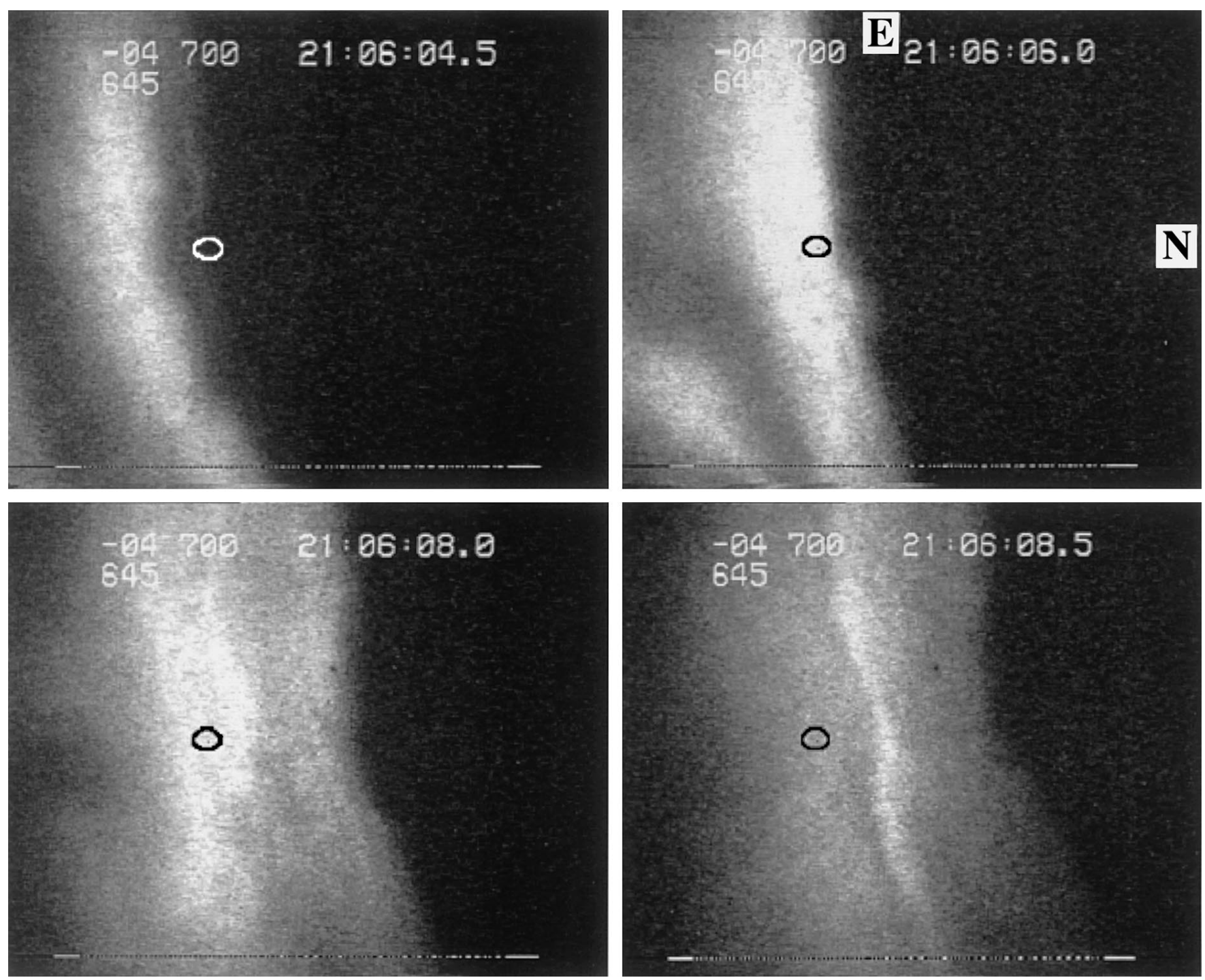

Fig. 3. Images from the narrow-angle TV camera at 21:06:04.5, 06.0, 08.0 and 08.5 UT. The position of the radar and photometer fields of view is marked. The field of view of the camera is $38 \mathrm{~km} \times 28 \mathrm{~km}$ at $100 \mathrm{~km}$, with north to the right and east at the top

When the field of view of the photometer is filled uniformly with auroral emission, the intensity of 427.8$\mathrm{nm}$ emission can be used as a measure of the input energy flux. A conversion factor of $220 \mathrm{R} / \mathrm{mW} \mathrm{m}^{-2}$ is adopted, which was derived from a set of electron transport calculations (Lummerzheim and Lilensten, 1994). As shown in the previous work, when the narrow bright filaments of the aurora do not fill the detector field of view, the energy flux as estimated from the emission rate is an average value. The energy flux calculated by the auroral model in that event was a factor of 10 larger, implying that the energy flux of $500 \mathrm{~mW} \mathrm{~m}^{-2}$ was precipitated in the narrow filament of $100 \mathrm{~m}$ width, about one-tenth of the area of the field of view. In the present case the same arguments can be applied and an estimate of the energy flux during the passage of the bright and narrow elements in the arc system has been made, using the same modelling techniques.

\subsection{Flux-first method}

To initialise the model, an estimate of the peak energy and energy flux was obtained from measured electron density profiles, using a 'flux-first' fitting algorithm (Palmer, 1995). In this method, an ionisation rate profile inferred from radar data is matched against libraries of model ionisation rate profiles, both Maxwellian and Gaussian (10\% width), computed with a neutral atmosphere appropriate for the given position and date. Varying peak energies and a downward energy flux of $1 \mathrm{~mW} \mathrm{~m}{ }^{-2}$ are adopted.

Figure 5 gives an overview of the energy flux in the radar field of view during the two passages of the arc system, obtained using this flux-first method. In order to obtain this result, the EISCAT electron density profiles have been converted to ionisation rate profiles, assuming a recombination rate coefficient $(\alpha)$. Although this is a gross approximation, it allows a very good estimate of 

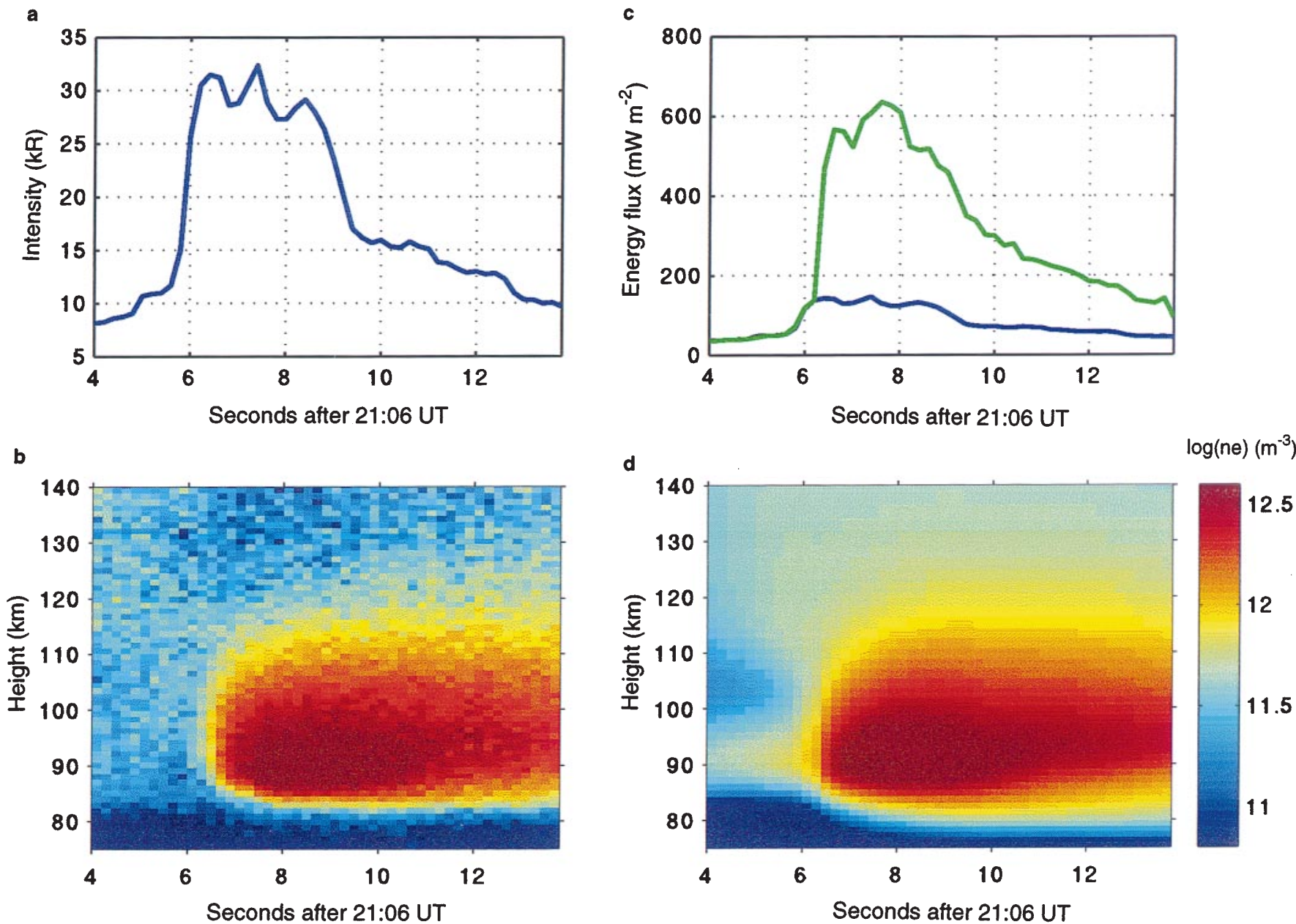

Fig. 4. a Measured 427.8-nm emission rate for $10 \mathrm{~s}$ during the second passage of the arc system. b Measured electron density at 0.2 -s resolution during second passage of arc system. c Blue curve is the

energy flux estimated from 427.8-nm emission rate as shown in a. Green curve is the energy flux required to produce the electron density profiles shown in $\mathbf{d}$, which match the measured profiles well

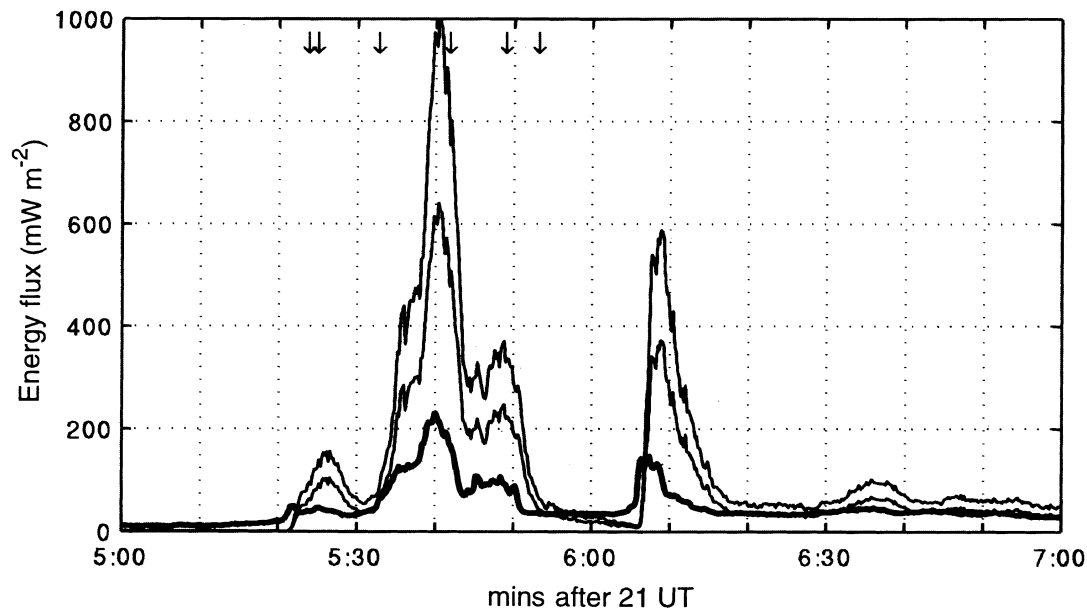

Fig. 5. Energy flux for 2-min interval covering both passages of the arc system. The thick line is estimated from the measured 427.8-nm intensity. The two thin lines are calculated with the 'fluxfirst' method described in the text, for two different assumed values of recombination rate coefficient $\alpha\left(2 \times 10^{-13}\right.$ and $\left.3 \times 10^{-13} \mathrm{~m}^{-3} \mathrm{~s}^{-1}\right)$. The larger value of flux results from the larger $\alpha$. The arrows on the top axis are the times of the profiles shown in Fig. 6

the peak energy of the precipitating flux, and yields reasonable limits for the energy flux. The thick line is the energy flux converted from the measured intensity of 427.8-nm emission. The other two lines result from different assumed values of $\alpha\left(2 \times 10^{-13}\right.$ and $3 \times 10^{-13}$ $\mathrm{m}^{-3} \mathrm{~s}^{-1}$ ) and show that there is a large difference at the times of increased activity, both between the measured and modelled, and between the different flux-first estimates. The larger value of flux results from the larger $\alpha$. Because $\alpha$ is height dependent (Nygrén et al., 1992), and the height of the peak precipitation is very variable through this interval, it is not reasonable to 
assume the same value throughout. However, this figure serves to show that very large fluxes, maybe in excess of $1 \mathrm{~W} \mathrm{~m} \mathrm{~m}^{-2}$, are precipitating in structures which are narrower than the radar beam. From this figure it is seen that the flux is in excess of that measured by the photometer whenever the aurora in the field of view is bright and structured, and at these times the larger value of $\alpha$ is appropriate. At times when bright, structured aurora is absent, the photometer-measured flux is appropriate most of the time, and the smaller value of $\alpha$ is a good estimate.

Another effect seen clearly in this representation is the time-delay between the arrival of the energetic precipitating particles in the beam, as demonstrated by the photometer increase (thick line), and the increase in electron density, from which the two thinner lines are derived (see 21:06:05 UT). The flux-first method must be used with care, remembering that the flux estimates are just that. Only the full, time-dependent model can account for the sudden changes that occur as electrons precipitate through the atmosphere. However, this method is fast and easy to use, and is able to shed much light on the changes seen in the measured profiles, before a full analysis is attempted. It also provides a good estimate of the flux magnitude, as the fitted profiles do not vary greatly from the measured profiles. One exception to this is seen at around 21:06:00 UT for the few seconds between the two passages of the arc system. This will be discussed in the following.

First passage of the arc system at 21:05:20 UT. The six panels of Fig. 6 demonstrate the results of the 'fluxfirst' fitting algorithm, in this case with $\alpha=3 \times 10^{-13}$ $\mathrm{m}^{-3} \mathrm{~s}^{-1}$. The times corresponding to these examples are marked with arrows on the top axis of Fig. 5. They are representative of the changes that are seen, and demonstrate how this method can give a first indication of the processes at work. Some ionisation rate profiles are well fitted by standard spectral shapes, others show a significant discrepancy. In each frame the thick line represents the best-fit monoenergetic (Gaussian) spectrum, and the thin line is the best-fit Maxwellian spectrum. These profiles will be referred to by the time in seconds after 21:05 UT, as shown on each panel.

In panels 1 and 2 (23.0 and 24.0) the first aurora has entered the radar beam and ionisation rates, derived directly from the measured electron density profiles, have increased from small to large values very suddenly. The increase in E-region ionisation rate is very narrow in height, and even a monoenergetic distribution is unable to account for this. This situation lasts for about $10 \mathrm{~s}$.

Panel 3 (31.6) corresponds to a slight lull in the aurora in the radar beam, and the distribution that fits best to the measured profiles has a Maxwellian shape.

Panel 4 (41.0) is at the time of maximum intensity of the aurora in the radar beam. A monoenergetic spectrum is a good fit.
Panel 5 (48.0) is an example of the need for more than one distribution to account for the ionisation produced. In this case the lower part of the profile is closer to a monoenergetic shape, and the top is more Maxwellian. A few seconds earlier the lower part of the profile was Maxwellian, with a distinct monoenergetic peak superimposed. This mixture of distributions persisted for about $10 \mathrm{~s}$.

In panel 6 (52.4) the inadequacy of one spectral shape is clear. There is an excess of ionisation above $115 \mathrm{~km}$ which cannot be accounted for by incoming precipitating flux. This remains the case for several seconds after this time with the profiles becoming distinctly double humped.

There are three categories seen in this sequence: profiles that are too narrow to be fitted (depleted electron density), those that exceed a standard fitted profile above some height, and those that fit well. The flux-first method with its approximations may be a limitation in some of these cases, and therefore a full model run is needed before drawing conclusions. The question that arises from this first analysis is whether the same processes in the atmosphere are responsible for the depleted profiles and for the excess density profiles, and next, what are these processes?

\subsection{Full model results}

Since there is no measured incident spectrum, it is necessary to estimate a spectral shape and total energy flux as input to this model. The method of combining Maxwellian and monoenergetic distributions is described in Lanchester et al. (1997). This is a very necessary part of the present analysis, as the profiles in Fig. 6 show that more than one distribution is present, and the optical images show clearly that the narrow elements do not fill the field of view. Only the second passage of the arc system is analysed here. Given the complexity of space and time variations, this event is much easier to unravel, since the 'arc' passes through the radar and photometer from $\mathrm{S}$ to $\mathrm{N}$ at a steady rate.

Second passage of arc system at 21:06:06 UT. Details of this passage of the double arc structure have been shown in the measurements of the narrow angle camera (Fig. 3), photometer (Fig. 4a) and radar (Fig. 4b). The results of a full model run for the same $10 \mathrm{~s}$ of data are shown in Fig. 4c,d. The comparison between the measured and modelled electron density profiles in panels $b$ and $d$ is very good, even though there are some significant differences. The input energy flux required to make such a good fit to the measured electron density is shown by the green line in panel c. This is the total energy flux, and is made up of two components, the Maxwellian background and the superimposed monoenergetic flux at the times of increased auroral precipitation. The blue line represents the flux derived from the 427.8-nm intensity (panel a). 

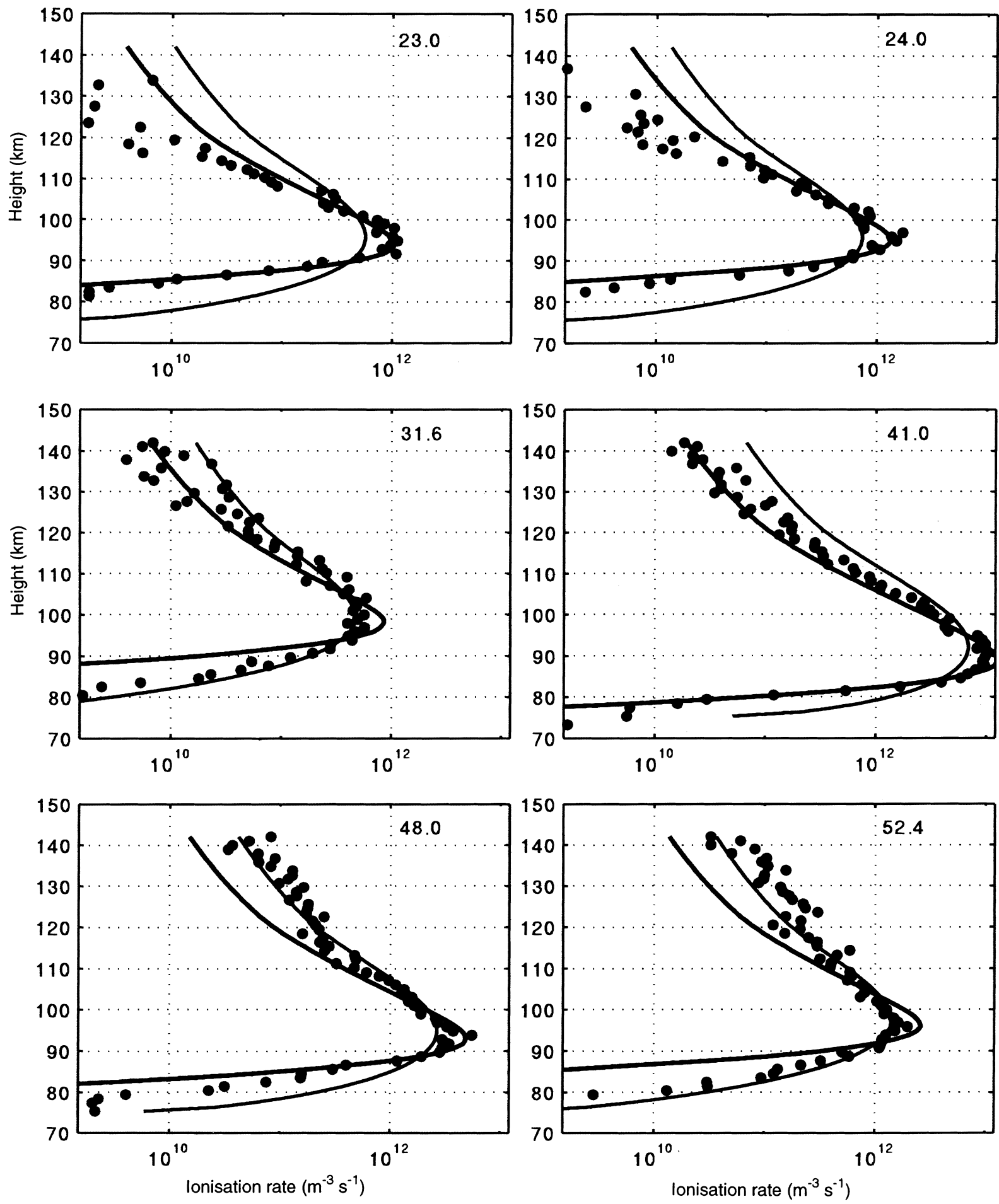

Fig. 6. Selected ionisation rate profiles from the first passage of the arc system. Black dots are from measurements at 0.2-s resolution. The thick line is a best fit to a monoenergetic input flux. The thin line is a

best fit to a Maxwellian input flux. These profiles show depletions and excess ionisation

In order to distinguish the physical differences between measured and modelled densities, height profiles at chosen times are given in Fig. 7. The first profile is at $4 \mathrm{~s}$ after 21:06 UT. (These profiles will be referred to by the time in seconds after 21:06 UT.) The dots are the measured electron densities at 0.2-s resolution. The solid line is the modelled density at

the same time resolution. In this first profile, the combination of the two spectral shapes can be seen, with the superimposed monoenergetic contribution peaking at $90 \mathrm{~km}$. The measured electron density profile is flatter than the modelled. This could be modelled more closely if two monoenergetic spectra with different peak energies, sharing the energy flux, 
21:06:4.0 UT

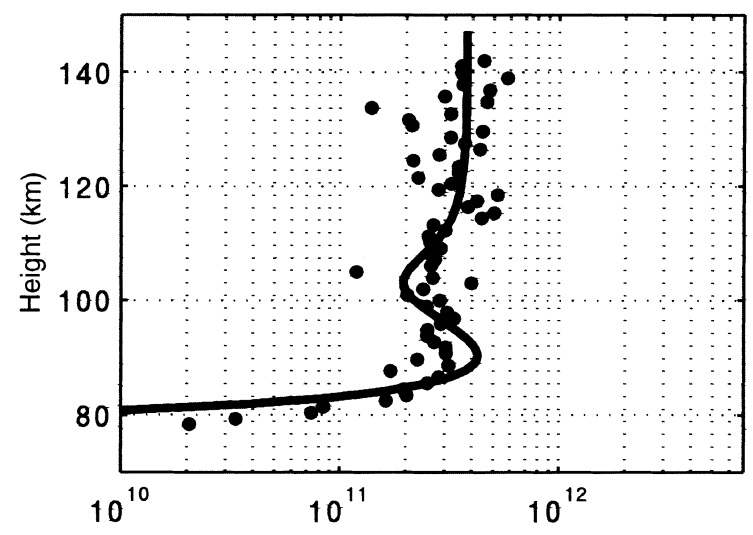

21:06:10.0 UT

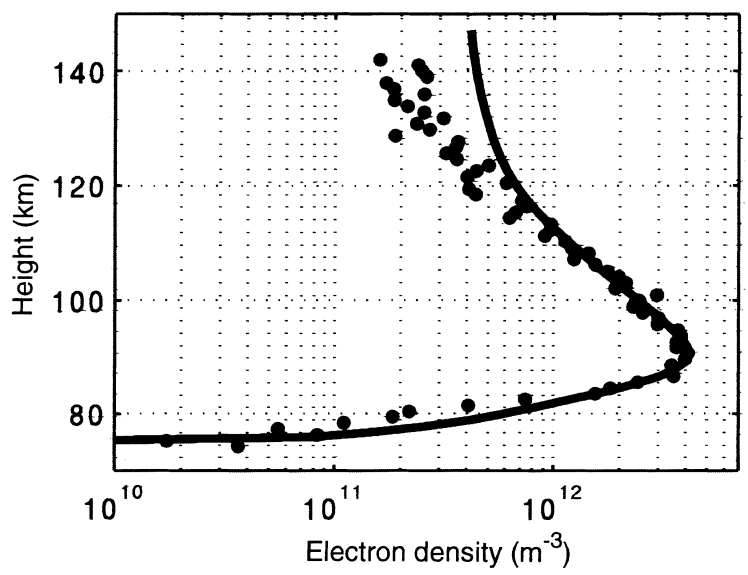

21:06:7.0 UT

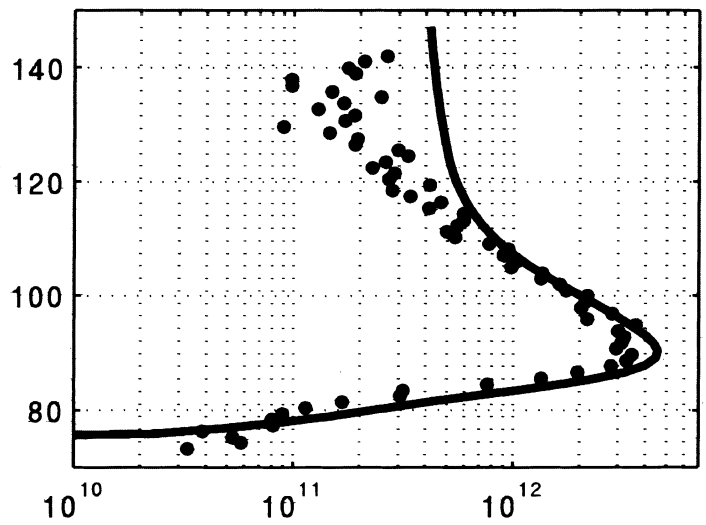

21:06:11.0 UT

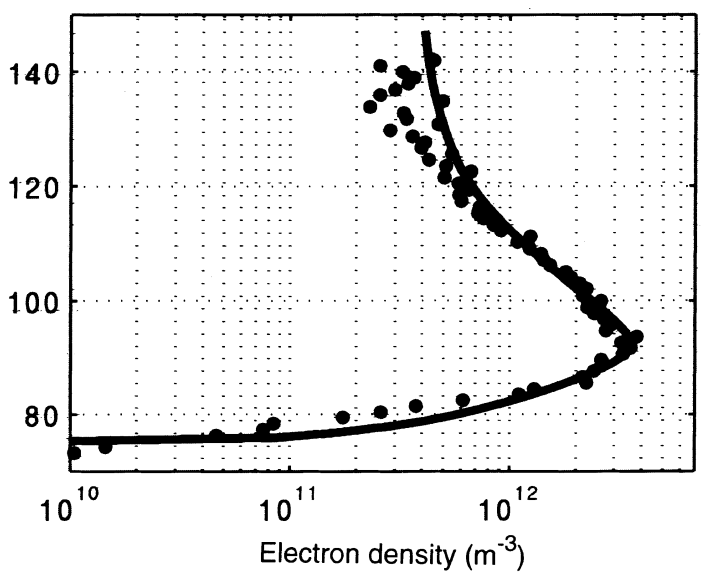

Fig. 7. Full model results of electron density profiles (line) and measured electron density profiles at 0.2-s resolution (dots)

were used, corresponding to two different filaments being sampled in the beam at the same time. This is not a relevant adjustment for the present purposes. The main feature to note in this panel is the total flux required to make a reasonable fit to the upper part of the profile. Referring to Fig. $4 \mathrm{c}$, the input flux at this time is equal to the flux measured by the photometer; so at the start of the model run the density is reproduced well by the measured flux.

The second profile shown in Fig. 7 is at $7 \mathrm{~s}$, after the arc has entered the photometer and radar beam. This profile is representative of all the profiles during the initial stages of the arc's presence in the beam. The measured densities are consistently below the values produced by the model, at heights above $115 \mathrm{~km}$. This is the same effect seen at the start of the first passage of the arc at 21:05:20 and lasts for several seconds. In that case the simple flux-first method could not fit a pure Gaussian distribution to the measured profiles. For the second passage, the full model, without the assumption of an effective recombination coefficient, also fails to fit the measured data. The effect is seen well in the colour panels of Fig. 4. The measured density has a cavity seen as a blue patch at $06-10 \mathrm{~s}$ above $120 \mathrm{~km}$. This is not reproduced by modelling. The third panel of Fig. 7 reinforces the result. The final panel of Fig. 7 shows the model making a better fit to the data. The cavity in electron density is being restored.

These four panels show that, as in the profiles of Fig. 6, using the flux-first fitting method, profiles of electron density are measured at the start of an auroral event that are too narrow in height to be fitted. It is these depleted profiles, rather than the excess profiles, that tell more about the physical processes that may be at work. It may be possible to reproduce bulges in profiles by adding flux to the low-energy contribution of the input spectrum, but 1D modelling cannot reproduce depleted profiles. It is possible that the bulges in profiles are produced by unusual precipitating distributions, but not density cavities.

\section{Discussion}

\subsection{Large electric fields}

In looking for a mechanism to explain the unusual density profiles, the large and variable electric fields would appear to be a contender. The direction of the electric field vectors is towards the bright arc as it approaches and recedes from the radar beam. The magnitude and changes in the fields are almost certainly 
even greater than those measured in a 3-s interval. The arc varies in position and brightness on time-scales much shorter than $3 \mathrm{~s}$. The electric field has great variability, with the largest measurement occurring during a short gap in the aurora in the radar beam, which is seen in the optical measurements, but not seen in the electron density measurements. Build-up and decay of the electron density do not match the very fast optical changes. It is therefore important to appreciate the possibility of large electric fields which appear to occur 'inside' auroral arcs when no optical measurements are available. The definition of an auroral arc is significant, and is a problem that has been highlighted in previous work of the present authors, and by other workers (Borovsky, 1993). The multiple structure of several parallel elements allows large fields to be measured within an arc system.

The wide-angle image of Fig 2 (third panel) at the time of the very large electric field $\left(600 \mathrm{mVm}^{-1}\right)$ demonstrates the difficulty of interpreting electric field measurements at high time resolution without the benefit of images. The field points towards the bright arc (SE) during a short time-interval when the aurora was not in the radar field of view. This gap in the aurora is not evident in the radar measurements of Fig. 1a, where the electron density is high throughout this event, but is seen in the photometer measurements of Fig. 1c. In this case the arc has moved in and out again from the radar beam within a few seconds. The largest field is measured beside the main structure, within about $10 \mathrm{~km}$. The energy flux in the narrow arc elements immediately prior to this large field measurement has been estimated at close to $1 \mathrm{~W} \mathrm{~m}^{-2}$ (Fig. 5). A fold in the length of the arc was seen at this time to correspond to an increase in the optical brightness. The large electric fields measured beside this structure reinforces the result of Lanchester et al. (1994) in which large and variable fields measured a few kilometres from a dynamic arc were associated with temporal changes. Only highresolution optical observations can unravel such events, and interpret them unambiguously. Both results indicate that the largest fields are measured a few kilometres from the arc, but that the temporal changes within the arc are of great importance.

Recent results from the FAST satellite and from earlier Freja measurements (Marklund et al., 1997) have shown that very large electric fields, of the same order of magnitude as measured here, are indeed part of the current systems close to arcs, and that there is often structure within these current systems, corresponding to very narrow regions of both upward and downward current. The horizontal electric fields are found to have reversals in direction over spatial scales of about $1 \mathrm{~km}$, which it is postulated correspond to diverging electric fields in the dark regions between arc elements (Marklund et al., 1994). The present results confirm that such large fields are present close to structured arc systems. Unfortunately the radar measurements are unable to match the satellite for temporal resolution, but the combination of optical measurements and radar improves the interpretation greatly.

\subsection{Effect on electron density profiles}

There are two distinct types of measured profiles displaying unusual shapes, those seen during the initial seconds of a bright auroral event, when the region around $115-120 \mathrm{~km}$ is depleted of ionisation, and those from the region close to an arc, either following its passage through the radar beam, or immediately preceding its arrival when there is excess ionisation. The depleted electron density profiles seen at 21:05:20 at the start of the first passage of the arc, and again at 21:06:07, correspond to a plasma scale height of 12 to $16 \mathrm{~km}$ which is unrealistically small, and are most likely the result of horizontal movement of plasma out of the radar field of view. Similarly, the bulges in electron density seen at the same height in the profiles from 21:05:50, between the two passages of the arc, are most likely caused by redistribution of plasma, resulting from the large velocities close to the arc boundaries.

Firstly, we consider the density profiles close to the arc. As already mentioned, the flux-first method does not do well at fitting the ionisation rate profiles in the interval between the two passages of the arc system (21:05:53 to 21:06:06 UT), estimating a flux that is lower than that measured by the photometer (Fig. 5). The full model run during this interval also has difficulty in reproducing the measured electron density profiles, which have a very distinct double-humped shape at the start of this interval, and become more and more flat in shape until the arrival of the arc. This is a significant clue to their being affected by electric fields, with horizontal movement of plasma into, then out of, the beam. After the start of the full model run, the input flux is kept at the value of the measured flux, estimated from the intensity of the 427.8-nm emission (see Fig. 4c). However, this flux is too great. The only way for the model to produce profiles as flat as those measured by the radar during the first few seconds of this run, is for the input flux to be smaller than that measured by more than $50 \%$.

The photometer measures emissions of $8 \mathrm{kR}$ throughout this interval, and the TV images show that there is a background emission. It is possible that some of the $427.8-\mathrm{nm}$ radiation was scattered into the field of view of the photometer from nearby bright features, and not produced by electron impact. Structure would not be seen during this interval because the cameras were both operating in a mode with very short exposure time. This gating is hand-controlled, and allows the contrast and dynamic range to be adjusted when very bright aurora is in the field of view. However, on this occasion the setting was not changed between the two passages of the arc. This would make observation of fainter structure difficult. Obviously there is hard precipitation present, as the electron density is of the order of $3 \times 10^{11} \mathrm{~m}^{-3}$ at heights as low as $85 \mathrm{~km}$ during this gap. In fact, as the arc moves into the field of view of the narrow-angle camera, extremely narrow bands appear for fractions of a second in front of the main arc. They cannot be reproduced well in this paper, but the remnant of one can possibly be seen in Fig. 3a. These 
bands resemble a twanging string attached to the main arc.

The other situation we find occurs when the extremely bright bands of aurora arrive in the radar beam. The modelled density profiles exceed the measured profiles above $115 \mathrm{~km}$. At this time the flux as measured by photometer is now several times too small to account for the peak electron density. As in Lanchester et al. (1997) this implies, and is borne out by the images from the narrow-angle camera, that energy resides almost entirely in sheets of precipitating monoenergetic electrons that are much narrower than the radar and photometer fields of view, i.e. in widths of $100 \mathrm{~s}$ of metres. But the shape of the profiles cannot be matched, even with a monoenergetic spectrum. We suggest that the electron densities are distorted by the large electric fields present near the arc edges. Plasma movement is occurring out of the arc elements, which have depleted profiles, into the region beside the arc, where the profiles exhibit bulges. The 1D model is not able to reproduce the event.

A simple 2D model has been put forward by Palmer (1995) to account for the observations already described. The model is based on the rotation of the mobility vector from predominantly perpendicular to the electric field vector in the F region, to predominantly parallel to it in the E region. Typical height profiles of the perpendicular (Hall) and parallel (Pedersen) mobilities with arbitrary scale are shown in Fig. 8 (adapted from Palmer, 1995). The ion velocity is given by $\mathbf{v}=\mathbf{k} \cdot \mathbf{E}$, where $\mathbf{k}$ is the mobility tensor and $\mathbf{E}$ is the electric field vector. The profiles indicate a transition in the ion velocity direction at about $120 \mathrm{~km}$, assuming that the electric field maps from the $\mathrm{F}$ to the $\mathrm{E}$ region, showing that plasma convection is not uniform with height. Both parallel and perpendicular mobilities decrease below about $110 \mathrm{~km}$, allowing little plasma convection. Above $115 \mathrm{~km}$ plasma could be transported in any direction, depending on the electric field vector, and not necessarily uniformly with height. Palmer (1995) has computed examples that readily match our observations.

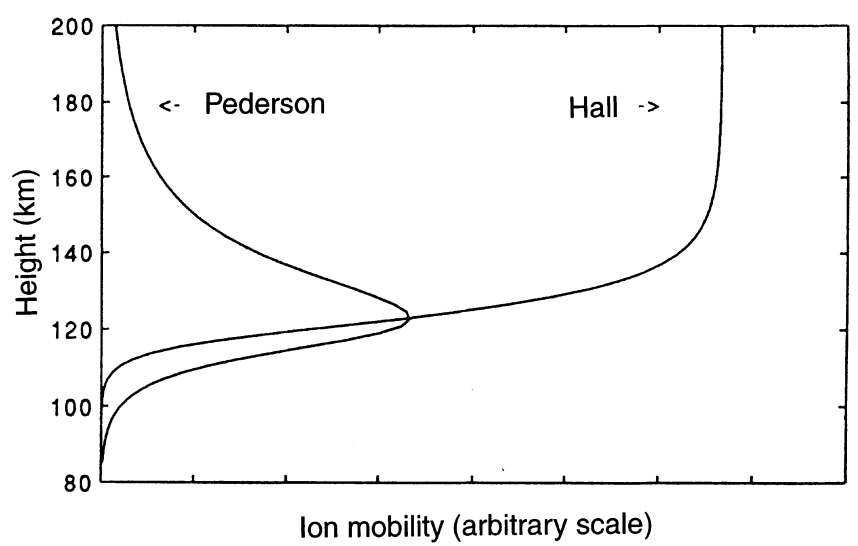

Fig. 8. Variation with height of Hall and Pedersen mobilities

\section{Summary}

The value of combining high-resolution optical measurements with incoherent-scatter radar measurements is stressed in this work. Interpretation of both radar and photometer measurements is dependent on modelling results, which combined with the spatial and temporal information from narrow-angle imagers, can take account of the fact that the fields of view of the instruments are not uniformly filled by auroral structures, and that mechanisms are at work in the ionosphere which affect the profiles of electron density. These factors are the response time of the ionosphere to extremely large and dynamic fluxes of electrons, and large electric fields found close to these precipitating sheets, which are capable of causing horizontal motion of the plasma.

Without the advantage of optical observations alongside radar measurements, the interpretation of the radar data is difficult. We have seen in the present results that very large electric fields are measured within regions of precipitation that appear continuous in the electron density measurements, but are in fact made up of separate auroral elements moving in and out of the radar beam. Optical measurements make clear the sequence of events, and in this case show that the large electric field is indeed in a region of no aurora. The radar-measured electron density is slow to respond to rapid changes. The initial rise in electron density lags the rise in emissions, and the subsequent variations are smeared out. The passage of the narrow and energetic filaments through the radar beam is seen in the images and reproduced in the photometer measurements.

Detailed modelling of electron density profiles produced by filamentary precipitation events has shown that ionisation, recombination and field-aligned diffusion cannot reproduce a variety of measured profiles. Large electric fields measured by the radar in association with the filamentary aurora can account for the distorted profiles, but a quantitative treatment requires multidimensional modelling. An elementary 2D model, suggested by Palmer (1995), provides a qualitative explanation of the radar observations.

While not the primary focus of this work, large electric fields and ion shear velocities may be responsible for the dynamic behaviour of the small-scale auroral features shown in Figs. 2 and 3. Candidate mechanisms that include ionosphere/magnetosphere coupling are the Kelvin-Helmholtz instability described in detail by Keskinen et al. (1988) and Keskinen and Ganguli (1996), and the 3D magnetic shear model of Otto and Birk (1991) described in some detail in Lanchester et al. (1997).

Acknowledgements. We thank the Director and staff of the EISCAT Scientific Association and the U.K. campaign team for help in running the EISCAT experiment. We thank Tauno Turunen for the design of the EISCAT experiment and E. Rieger, H. Loidl and H. Holma for their contribution to the optical measurements. Henry Rishbeth and Li Zou helped in discussions and practical matters. BSL is supported by a grant from the 
PPARC in the UK. Work by MHR is supported by the NSF grant ATM94-09485. The EISCAT Scientific association is supported by the Suomen Akatemia of Finland, Centre National de la Recherche Scientifique of France, Max-Planck Gesellchaft of the Federal Republic of Germany, Norges Almenvitenskapelige Forskningsråd of Norway, Naturvetenskapliga Forskningsrådet of Sweden and the Particle Physics and Astronomy Research Council of the United Kingdom.

The Editor-in-Chief thanks M. J. Keskinen and G. Marklund for their help in evaluating this paper.

\section{References}

Boehm, M. H., G. Paschmann, J. Clemmons, G. Haerendel, L. Eliasson, and R. Lundin, Freja observations of narrow inverted$\mathrm{V}$ electron precipitation by the two-dimensional electron spectrometer, Geophys. Res. Lett., 21, 1895-1898, 1994.

Boehm, M. H., J. Clemmons, and G. Paschmann, Freja observations of a ten-meter boundary within monoenergetic auroral electron precipitation, Geophys. Res. Lett., 22, 69-72, 1995.

Borovsky, J. E., Auroral arc thicknesses as predicted by various theories, J. Geophys. Res., 98, 6101-6138, 1993.

Carlson, C. W., J. McFadden, R. Ergun, W. Peria, D. Klumpar, and R. Elphic, Measurements of trapped ion conics associated with upgoing electron beams and diverging electrostatic shocks, Trans. Am. Geophys. Un., 78, 605, 1997.

Frey, H. U., W. Lieb, O. H. Bauer, H. Höfner, and G. Haerendel, CCD-camera system for stereoscopic optical observations of the aurora, SPIE Proc., 2863, 1996.

Kaila, K. U., and R. A. Rasinkangas, Coordinated photometer and incoherent scatter radar measurements of pulsating arcs with high time resolution, planet, Space Sci., 37, 545-553, 1989.

Keskinen, M. J., and Ganguli, Simulation of dynamics and structure in the black aurora, J. Geophys. Res., 101, 2699526999, 1996.
Keskinen, M. J., H. G. Mitchell, J. A. Fedder, P. Satyanarayana, S. T. Zalesak, and J. D. Huba, Nonlinear evolution of the KelvinHelmholtz instability in the high-latitude ionosphere, J. Geophys. Res., 93, 137-152, 1988.

Lanchester, B. S., J. R. Palmer, M. H. Rees, D. Lummerzheim, K. U. Kaila, and T. Turunen, Energy flux and characteristic energy of an elemental auroral structure, Geophys. Res. Lett., 21, 27892792, 1994.

Lanchester, B. S., K. Kaila, and I. W. McCrea, Relationship between large horizontal electric fields and auroral arc elements, J. Geophys. Res., 101, 5075-5084, 1996.

Lanchester, B. S., M. H. Rees, D. Lummerzheim, A. Otto, H. U. Frey, and K. U. Kaila, Large fluxes of auroral electrons in filaments of $100 \mathrm{~m}$ width, J. Geophys. Res., 102, 9741-9748, 1997.

Lummerzheim, D., and J. Lilensten, Electron transport and energy degradation in the ionosphere: evaluation of the numerical solution, comparison with laboratory experiments and auroral observations, Ann. Geophysicae, 12, 1039-1051, 1994.

Marklund, G. T., L. Blomberg, C.-G. Falthammar, and P.-A. Lindqvist, On intense diverging electric fields associated with black aurora, Geophys. Res. Lett., 21, 1859-1862, 1994.

Marklund, G. T., T. Karlsson, and J. Clemmons, On low-altitude particle acceleration and intense electric fields and their relationship to black aurora, J. Geophys. Res., 102, 17509$17522,1997$.

Nygrén, T., K. U. Kaila, and A. Huuskonen, Determination of Eregion effective recombination coefficient using impulsive precipitation events, Geophys. Res. Lett., 19, 445-448, 1992.

Otto, A., and G. T. Birk, Formation of thin auroral arcs by current striation, Geophys. Res. Lett., 20, 2833-2386, 1993.

Palmer, J.R., Plasma density variations in the aurora, PhD thesis, Univ. of Southampton, 1995.

Rasinkangas, R. A., K. U. Kaila, and T. Turunen, Comparison of the lower border of aurorae determined by two optical emission ratio models, Planet. Space Sci., 37, 1117-1126, 1989. 